\title{
GPPS-BJ-2019-0170
}

\section{Application of Stall Warning Approach with Stall Precursor Suppressed Casing Treatment on a Two-Stage Compressor}

\author{
Ruize Xu \\ Beihang University \\ xuruize@buaa.edu.cn \\ Beijing, China
}

\author{
Xu Dong \\ Beihang University \\ buaadongxu@buaa.edu.cn \\ Beijing, China
}

\author{
Jia Li \\ Beihang University \\ brucelee@buaa.edu.cn \\ Beijing, China
}

\author{
Dakun Sun \\ Beihang University \\ sundk@buaa.edu.cn \\ Beijing, China
}

\author{
Xiaofeng Sun \\ Beihang University \\ sunxf@buaa.edu.cn \\ Beijing, China
}

\begin{abstract}
A stall-warning approach based on aero-acoustic theory is studied in this paper. For this stall warning approach, a parameter $\mathrm{Rc}$ is defined to measure the periodicity of the blade-passing signal. Signal simulation is used to investigate the mechanism of the stall warning approach. The results suggest that the value of Rc is influenced by the power of the perturbance. The experiments on a 2-stage compressor indicate this stall warning approach can generate a warning signal several seconds before the stall. It is demonstrated in this paper that the stall warning approach can detect the distribution and evolution of stall precursors. According to the distribution of the stall precursors, the partial SPS casing treatment is applied and realized a stabilization of compressor.
\end{abstract}

\section{INTRODUCTION}

Rotating stall and surge are two aerodynamic instabilities in aero-engines which restrict the performance of the compression system. To ensure that an engine could have a stable performance in a variety of working conditions such as inlet distortions, speed transients, thermal transients, and engine control tolerances, it is common to require the design operating point of the compressor to have about $20 \%$ stall margin. In some cases, that designs should sacrifice some efficiencies to achieve the stall margin requirement. One way to expand the stable operating range of compressors is to make use of control technologies. Active control of compressor instabilities was originally proposed by Epstein et al. [1] in 1989. They advocated a strategy to suppress the onset of stall and surge by controlling the stall inception. Then Ffowcs Williams and Huang [2] firstly realized active surge suppression on a centrifugal compressor in the laboratory. Since then plenty of investigations were conducted to detect and study stall inception using the relevant signal processing of Fourier analysis [3-5], wavelet techniques [6-8]. These techniques are very useful for analyzing the stall mechanism. In some compressors, the stall inception would evolve to full developed rotating stall within several rotor revolutions, which means that the evolution time is too short for a control system to realize stall margin enhancement for practical. Thus, how to get a longer warning time ahead of rotating stall inception is a problem have to be solved.

Inoue et al. [9] conducted a series of experiments on a low-speed axial compressor and confirmed that the pressure signal of passing blades was periodical and the collapse of the periodicity could be used to detect the stall precursor. They also proposed a parameter called similarity coefficient to describe the periodicity of the blade-passing signal. This similarity coefficient needs more than 200 rotor revolutions sampling data and thus it is not appropriate for the transient condition. Tahara et al. [10, 11] used the auto-correlation coefficient calculated from the pressure signals of the current and one rotor revolution before of the identical blades and studied the factors that had influences on the value of the coefficient. Tests on a low-speed compressor showed that this method had the potential to generate a stall warning signal in advance of stall inception. Dhingra et al. [12,13] suggested a stall detecting technique using correlation measure to evaluate the irregularity of the pressure signal from sensors over the rotor and developed a stochastic model of the correlation measure. After analysing the data from both low-speed and high-speed compressors, they defined the correlation measure dropped below a given threshold as an "event" and found time between events decreases as flow rate close to the stall boundary. Young et al. [14] did some further investigation of the correlation measure on a low-speed compressor and found the value of the correlation measure was strongly affected by both tip-clearance size and eccentricity.

All these researches above utilized the deterioration of blade-passing periodicity near stall but did not have enough 
theoretical analysing for the mechanism behind this phenomenon. Li et al. [15] considered the collapse of periodicity could reflect the evolution of stall precursor. From this perspective, they explained the mechanism of this phenomenon by using aero-acoustic theory and vortex theory. Li considered that the evolution of stall precursor was always accompanied by complex vortex-like flow separation and vortex shedding on the blade. Those vortex flow which could influence the blade circulation caused the collapse of periodicity. Based on this theory, Dong et al. [16] applied this stall warning technique on a kind of stall precursor-suppressed (SPS) casing treatment to realize an online control. The mechanism of SPS casing treatment is to affect the evolution of the stall precursors in order to obtain stall margin improvement [17-19], and according to experimental results on low-speed and high-speed compressors, this SPS casing treatment can enhance the stall margin by $8 \%-18 \%[17,18$, 20]. The demonstration on a single-stage low-speed compressor showed that this online control method could effectively enlarge the working range of compressor [16].

It should be noticed that the study of this stall warning approach still remains on the single-stage compressor. When it comes to multi-stage compressor, flow structure becomes much more complicated, so in this paper we study how this stall warning technique performs in a multi-stage compressor and investigate the influence of transducer position on this technique. The paper is structured as below: firstly, the basic idea of the stall warning approach is introduced, and a series of sinusoidal signals are chosen to simulate the blade signals and the stall warning approach is used to dispose these signals to study how different perturbations influence the results of this approach and to investigate the mechanism of the approach. Then this technique is implemented on a two-stage compressor to study the feature of the warning time. Next, sensors are installed on the different positions of the compressor to study the distribution and evolution of stall perturbations in the compressor. Then SPS casing treatment is used to realize a local control of precursor and enlarge the stable working range of the compressor.

\section{EXPERIMENTAL FACILITIES}

\section{The two stage compressor}

The experiments were performed on a two-stage lowspeed axial compressor with inlet guide vane. The two-stage compressor shown in Fig.1 contains an inlet duct, an inlet guide vane (IGV), two rotor-stator stages, an outlet duct and a throttle comprising of a fixed cone and movable annular sleeve. The rotating speed of this compressor can be varied from 0 to $3000 \mathrm{rpm}$. The basic design parameters of the compressor are listed in table 1 .

\section{Measurement system}

In the experiments, the measurement system contains steady characteristic measurement and dynamic pressure measurement. The steady measurement consists of total pressure sensors in the inlet and outlet duct and the static pressure sensors placed on the casing wall of the inlet and outlet duct to get the characteristic parameters of the compressor (Fig.1). High-frequency Kulite pressure sensors are mounted on the casing and flushed with the inner wall, the measurements are adopted at the cross sections of leading edge of IGV, the first stage rotor and the second stage rotor respectively (as shown in Fig.2) to capture the unsteady pressure signal of passing blades. The Sampling rate of the transducers is $100 \mathrm{KHz}$. Considering the blade passing frequency $(\mathrm{BPF})$ is approximately $2.4 \mathrm{KHz}$. This sampling rate can meet the requirement for unsteady pressure measurement. In the experiment, the phase-locking technique is also implied to help compare the blade passing signal of two contiguous revolutions. This technique uses a Hall sensor fixed on the shaft to give a pulse signal at a certain circumferential position in each revolution. The configuration of these transducers is displayed in fig. 2 . 


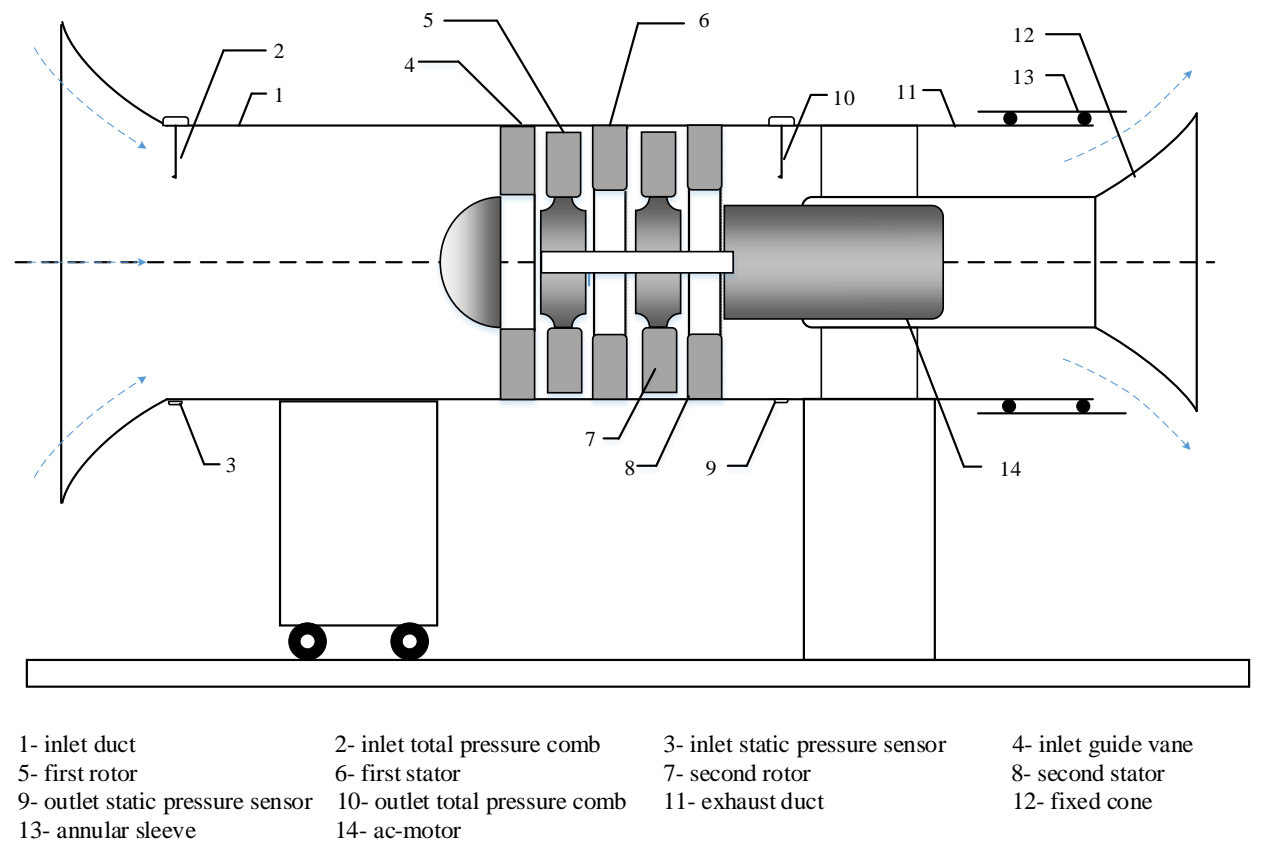

Fig.1 Configuration of the 2-stage compressor

Table 1 Design Parameters of the two-stage compressor

\begin{tabular}{cccccc}
\hline & \multicolumn{2}{c}{ Geometrical Parameters } & & \\
\hline \hline & IGV & $\begin{array}{c}1^{\text {st }} \\
\text { Rotor }\end{array}$ & $\begin{array}{c}\mathbf{1}^{\text {st }} \\
\text { Stator }\end{array}$ & $\begin{array}{c}\mathbf{2}^{\text {nd }} \\
\text { Rotor }\end{array}$ & $\begin{array}{c}\mathbf{2}^{\text {nd }} \\
\text { Stator }\end{array}$ \\
\hline Blade Number & 38 & 47 & 45 & 47 & 45 \\
\hline Established Angle & $0^{\circ}$ & $60^{\circ}$ & $10^{\circ}$ & $60^{\circ}$ & $10^{\circ}$ \\
\hline $\begin{array}{c}\text { External } \\
\text { Diameter/mm }\end{array}$ & 600 & 600 & 600 & 600 & 600 \\
\hline Span-chord Ratio & 1.69 & 1.69 & 1.69 & 1.69 & 1.69 \\
\hline Hub-tip Ratio & 0.7 & 0.7 & 0.7 & 0.7 & 0.7 \\
\hline \hline Design Rotating Speed & Aerodynamic Parameter & Mass flow & & $6.8 \mathrm{~kg} \cdot \mathrm{s}^{-1}$ \\
\hline Total pressure ratio & 3000rpm & Pressure rise & & $4900 \mathrm{~Pa}$ \\
\hline \hline
\end{tabular}

\section{STALL WARNING APPROACH}

In a compressor, it is clear that the blade-passing signal of two neighbouring shaft periods is similar, and this similarity will degrade with the reducing of stall margin. Fig. 3 displays the time-pressure signals of the 2-stage compressor sampled by the sensor located on the casing in front of the IGV. The red and black curves are the signals of the current and previous shaft period, respectively. At the design point, the current signal is almost accordant with the signal one shaft period before. At the near stall point, because the flow separation and vortex shedding occur more frequently, which affect the circulation of blades, the pressure signals of two contiguous revolutions are quite different from each other [15].

A parameter called $\mathrm{Rc}$ is used to indicate the periodicity of the pressure signal, which can be calculated via the correlation measure as follows:

$$
R c(j)=\frac{\vec{p}_{j}^{n} \cdot \vec{p}_{j-N}^{n}}{\left|\vec{p}_{j}^{n}\right| \cdot\left|\vec{p}_{j-N}^{n}\right|}
$$

Where $R c(j)$ is the value of $R c$ at present sample point $j$. The calculation process of the correlation measure is illustrated in Fig. 4. Vector $\overrightarrow{\vec{p}_{J}^{n}}$ is the pressure signal. $\mathrm{N}$ is the number of samples in one shaft revolution and $n$ equals to the number of samples in a calculating window. In this paper, $\mathrm{n}$ is set as the number of 3 blade samples. The reason for choosing 
3 blades will be explained in the next section. By its mathematical definition, the range of $\mathrm{Rc}$ is from -1 to 1 , and

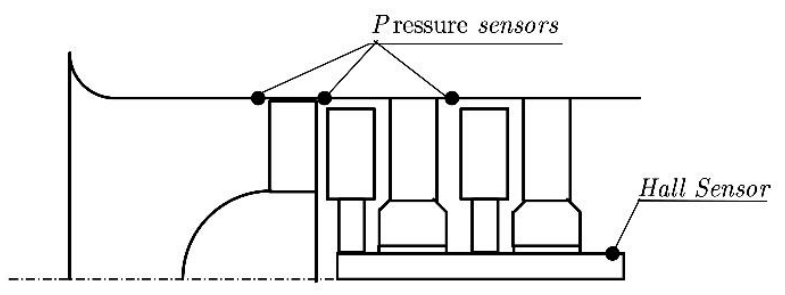

the closer this value is to one, the better the signal periodicity is.

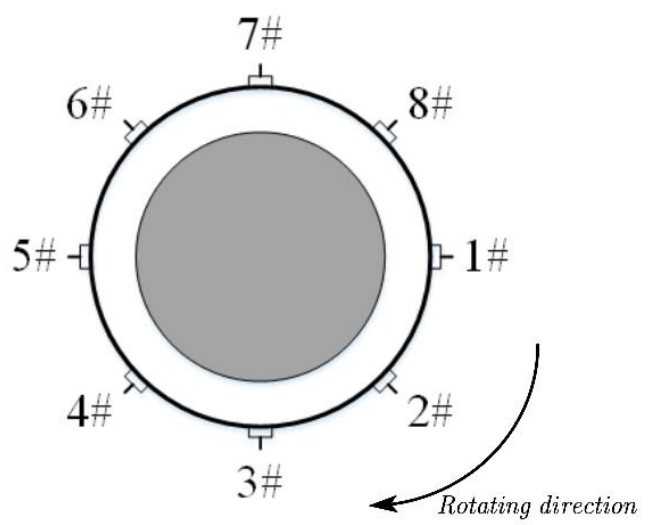

Fig. 2 Configuration of the transducers

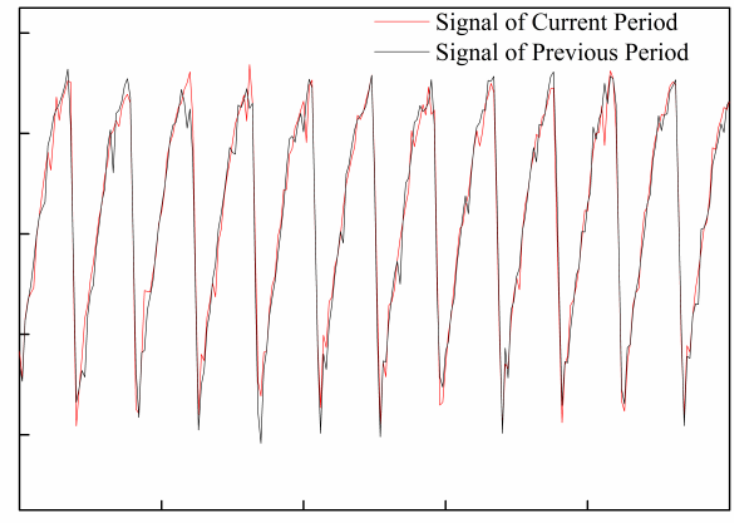

(a) At design point

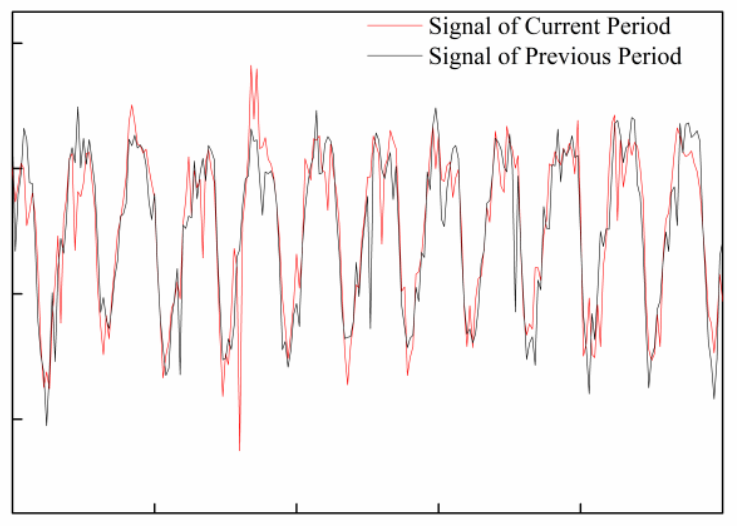

(b) Near stall point

Fig.3 Pressure signal of two neighboring shaft period

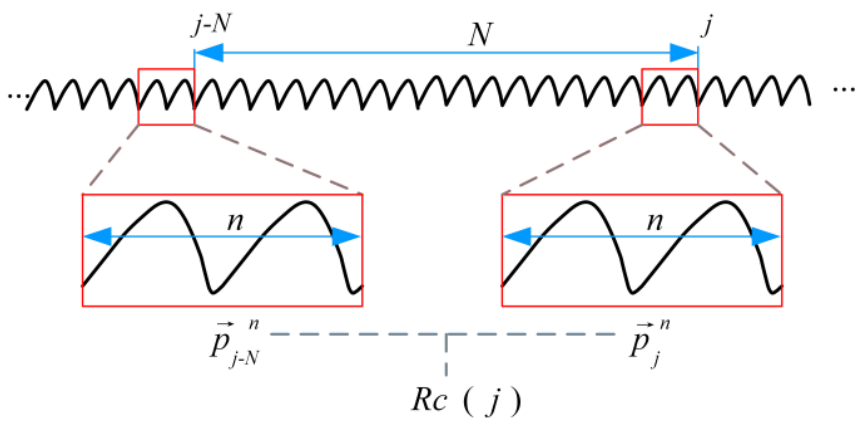

Fig.4 Calculation process of correlation measure

\section{Signal simulation}

According to the Fourier transform, a complicated signal can be represented by the sum of sine signals with different frequency, so we select sine signals with some certain frequency to simulate the blade signals of the two-stage compressor in order to investigate how different perturbance influence result of correlation measure and explain the mechanism of this stall warning approach.

As mentioned above, the rotation frequency of the shaft $\left(f_{s}\right)$ is $50 \mathrm{~Hz}$, and the blade number of the first rotor is 47 . Thus, the blade passing frequency (BPF) is $2350 \mathrm{~Hz}$. Ignoring the blade-to-blade difference, we describe the steady blade signal as:

$$
p_{0}=a_{0} \sin (4700 \pi t)
$$

In axial flow compressors, there are two typical low frequency perturbations appear before the stall occurs: "modal wave" and "spike". The "modal wave" rotates at a relatively low speed below $50 \%$ of rotor speed $\left(f_{s}\right)$, and "spike" propagates more quickly at speeds between $70 \%$ and $90 \%$ of the rotor speed $\left(\mathrm{f}_{\mathrm{s}}\right)$. According to the rotating speed of the "modal wave" and "spike", $0.5 \mathrm{f}_{\mathrm{s}}(25 \mathrm{~Hz})$ and $0.9 \mathrm{f}_{\mathrm{s}}(45 \mathrm{~Hz})$ signals are chosen to represent the low frequency perturbations in the compressor. Due to the interaction effect between two adjacent blade rows, there are some perturbations 
whose frequencies are close to multiples of the BPF, so the signals with frequencies of $94.5 \mathrm{f}_{\mathrm{s}}(4725 \mathrm{~Hz})$ and $94.9 \mathrm{f}_{\mathrm{s}}$ $(4745 \mathrm{~Hz})$ are selected to stand for the high frequency perturbations in the compressor. Here, four signals whose frequencies are $0.5 \mathrm{f}_{\mathrm{s}}(25 \mathrm{~Hz}), 0.9 \mathrm{f}_{\mathrm{s}}(45 \mathrm{~Hz}), 94.5 \mathrm{f}_{\mathrm{s}}(4725 \mathrm{~Hz})$ and $94.9 \mathrm{f}_{\mathrm{s}}(4745 \mathrm{~Hz})$ are added into the steady blade signal to represent four different perturbations. The expressions of these four signals are

$$
\begin{aligned}
& p_{1}=a_{1} \sin (50 \pi t) \\
& p_{2}=a_{2} \sin (90 \pi t) \\
& p_{3}=a_{3} \sin (9450 \pi t) \\
& p_{4}=a_{4} \sin (9490 \pi t)
\end{aligned}
$$

Compared with the steady blade-passing signal, the power of perturbance in a compressor is rather small, so the amplitude of perturbance $a_{i}$ is set equal to $0.1 a_{0}$ and $0.3 a_{0}$ for each perturbance with different frequency.

Fig.5 depicts the Rc value of the steady blade-passing signal. In this figure, the value of Rc keeps at 1 from beginning to the end, which indicates a perfect periodicity of the bladepassing signal at the design point of the compressor. Fig.6-9 depicts the correlation measure results of the signal $p_{0}$ with different perturbance $p_{1}, p_{2}, p_{3}$ and $p_{4}$. The mean value of Rc for signals with each perturbance with different amplitude is detailed in Table 2.

\begin{tabular}{|c|c|c|c|c|}
\hline signals & $a_{i}=0.1 a_{0}$ & Range of Rc & $a_{i}=0.3 a_{0}$ & Range of Rc \\
\hline$p_{0}+p_{1}$ & 0.980 & $0.961-1$ & 0.841 & $0.695-1$ \\
\hline$p_{0}+p_{2}$ & 0.998 & $0.996-1$ & 0.984 & $0.966-1$ \\
\hline$p_{0}+p_{3}$ & 0.980 & $0.980-0.980$ & 0.835 & $0.834-0.836$ \\
\hline$p_{0}+p_{4}$ & 0.998 & 0.998-0.998 & 0.984 & 0.983-0.985 \\
\hline
\end{tabular}

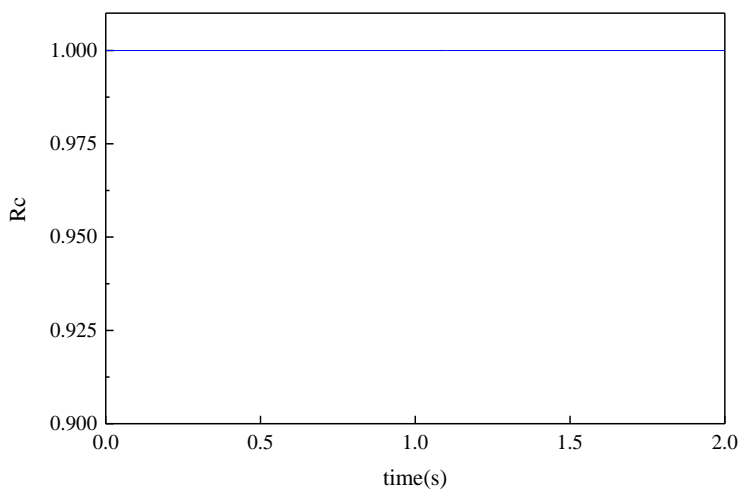

Fig. 5 Rc of blade-passing signal

Table 2 Mean value of Rc for signals with different perturbance

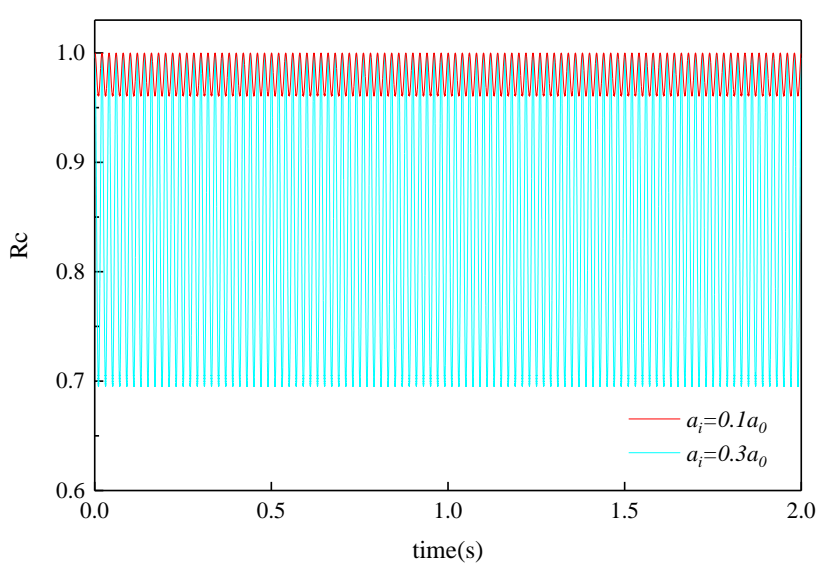

Fig. 6 Rc of blade-passing signal with $25 \mathrm{~Hz}$ perturbance

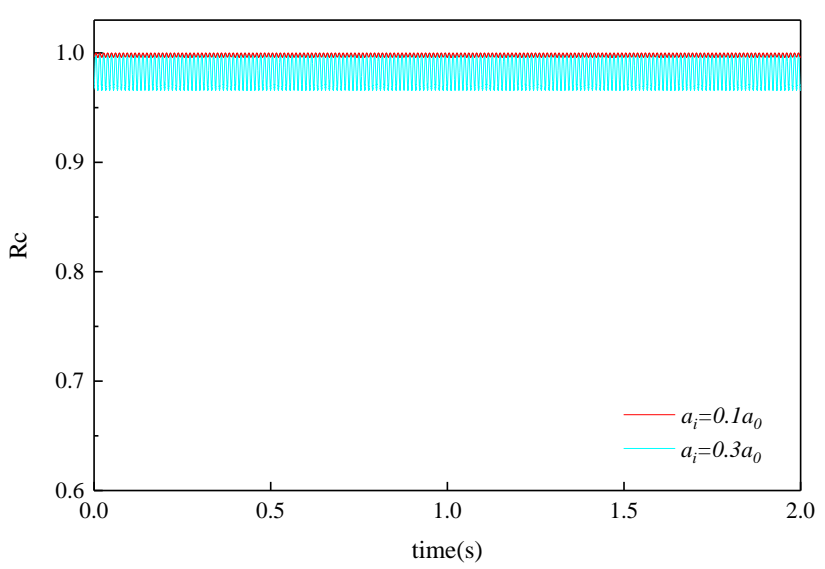

Fig. 7 Rc of blade-passing signal with $45 \mathrm{~Hz}$ perturbance 


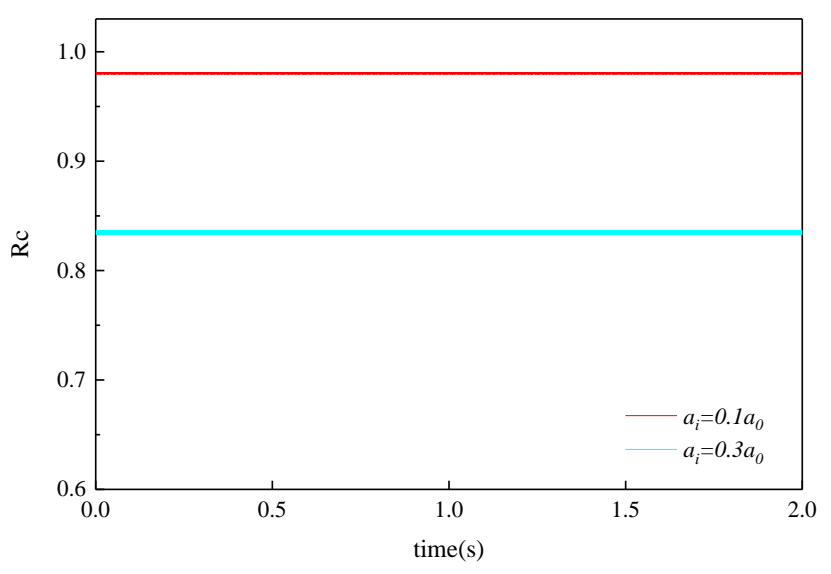

Fig. 8 Rc of blade-passing signal with $4725 \mathrm{~Hz}$ perturbance

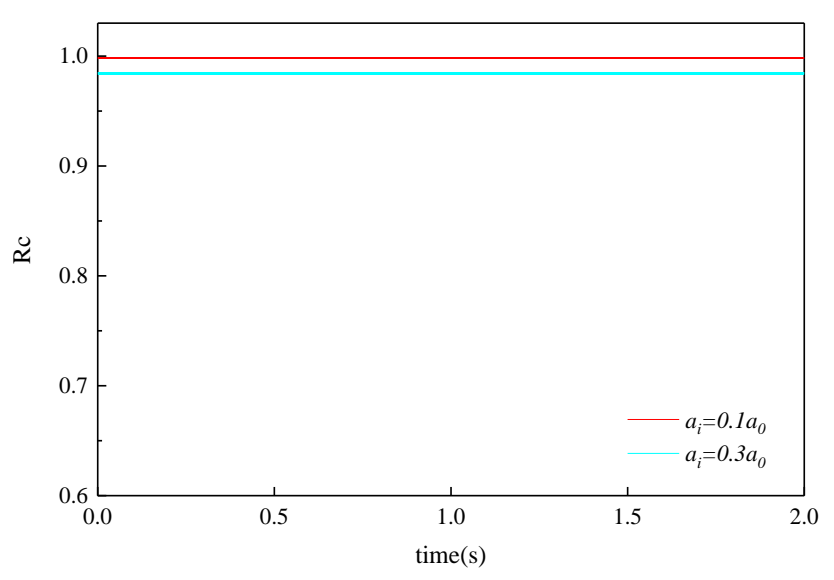

Fig. 9 Rc of blade-passing signal with $4745 \mathrm{~Hz}$
perturbance

It can be identified in table 2 that, when the amplitude of perturbance is $0.1 a_{0}$, the mean value of Rc for signals with different perturbance is $0.980,0.998,0.980$ and 0.998 , and if the amplitude increases to $0.3 a_{0}$, it decreases to 0.841 , $0.984,0.835$ and 0.984 . This result indicates that for signals with a certain frequency perturbance if the amplitude of perturbance is amplified, the mean value of Rc will decrease with verifying degrees.

As evident from table 2, the correlation measure results of signal $p_{0}+p_{2}$ and $p_{0}+p_{4}$ are the same, and the mean values of $\mathrm{Rc}$ for signal $p_{0}+p_{1}$ and $p_{0}+p_{3}$ are also highly identical. It should be noticed that the frequency differences between $p_{1}, p_{3}$ and $p_{2}, p_{4}$ are both $4700 \mathrm{~Hz}$. This suggests that two perturbance between which the difference of frequency is a certain value may have a similar influence on the mean value of Rc. For a certain perturbance $p_{i}$, its frequency $f_{i}$ can be expressed as $f_{i}=\left(x_{i}+y_{i}\right) f_{s}, x_{i} \in N, y_{i} \in[0,1)$. The correlation measure compares the blade-passing signals of two adjacent revolutions. The signal $p_{0}+p_{i}$ at present and one revolution before can be written as

$$
\begin{aligned}
\left(p_{0}+p_{i}\right)_{\text {now }} & =a_{0} \sin \left(4700 \pi t_{0}\right)+a_{i} \sin \left[2 \pi\left(x_{i}+y_{i}\right) f_{s} t_{0}\right] \\
& =a_{0} \sin \left(4700 \pi t_{0}\right)+a_{i} \sin \left(2 \pi f_{i} t_{0}\right) \\
\left(p_{0}+p_{i}\right)_{\text {rev }}= & a_{0} \sin \left[4700 \pi\left(t_{0}-\frac{1}{f_{s}}\right)\right]+a_{i} \sin \left[2 \pi\left(x_{i}+y_{i}\right) f_{s}\left(t_{0}-\frac{1}{f_{s}}\right)\right] \\
= & a_{0} \sin \left(4700 \pi t_{0}\right)+a_{i} \sin \left(2 \pi f_{i} t_{o}-2 \pi y_{i} f_{s}\right)
\end{aligned}
$$

Comparing two equations shows that $x_{i}$ has no influence to the difference between two signals, which indicates if the difference of frequency between two perturbance is equal to the integral multiple of shaft frequency this two perturbance will have an identical effect on the mean value of Rc. According to the periodicity property of sine signals, perturbance whose $y_{i}$ is close to 0 or 1 brings fewer differences to signals of two adjacent revolutions than perturbance of which the value of $y_{i}$ is near 0.5 does. This explains why the mean value of $\mathrm{Rc}$ for $p_{0}+p_{2}$ and $p_{0}+p_{4}$ is obviously higher than $p_{0}+p_{1}$ and $p_{0}+p_{3}$.

Although the mean value of $\mathrm{Rc}$ for $p_{0}+p_{1}$ and $p_{0}+p_{3}$ is almost equal. The range of Rc in Fig.6 is significantly larger than the Rc in Fig.8. This difference in Rc range can be attributed to the short length of the calculating window. As can be seen in the Fig.10, when the scale of calculating window is rather smaller than the perturbance wavelength, the influence that perturbance brings to the signal changes distinctly with time. In this figure, the $\mathrm{Rc}$ at $t_{1}$ is lower than the Rc at time $t_{2}$. If the calculating window is extended from 3 blades to 47 blades (one revolution), the range of $\mathrm{Rc}$ for $p_{0}+p_{1}$ and $p_{0}+p_{3}$ is almost identical (Fig.11). According to reference [15], in a compressor working at near stall point, the perturbance mainly consists of flow separation and vortex shedding on the blade. The scale of these flow phenomenon usually covers two to three blades, so a calculating window covering three blades is an appropriate choice. Hence, the distribution of Rc can be used to distinguish the low and high frequency perturbance for the range of Rc for signals with low frequency perturbance is large whereas for signals with high frequency perturbance it is small.

Thus, the mechanism of this stall warning approach can be explained as below. Considering that the unsteadiness of flow separation and vortex shedding, the frequency components of the perturbance are complicated and when the operating point of a compressor is slowly driven to stall boundary, the power of the perturbance will grow and the value of Rc will decrease. 


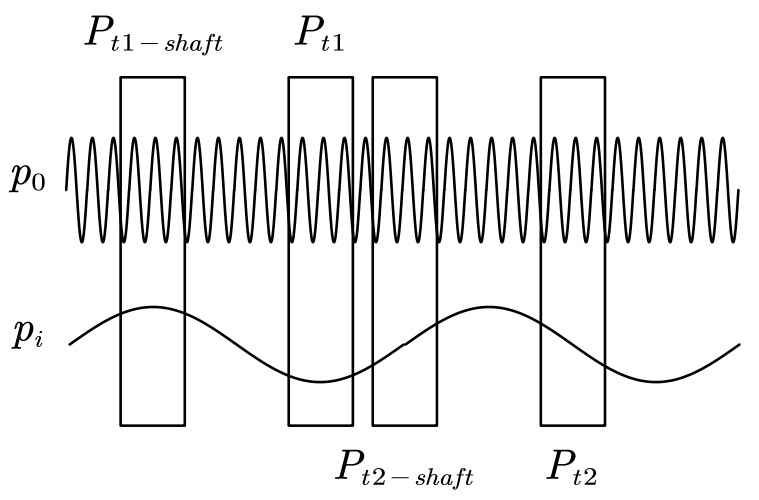

Fig. 10 Influence of perturbance at different time

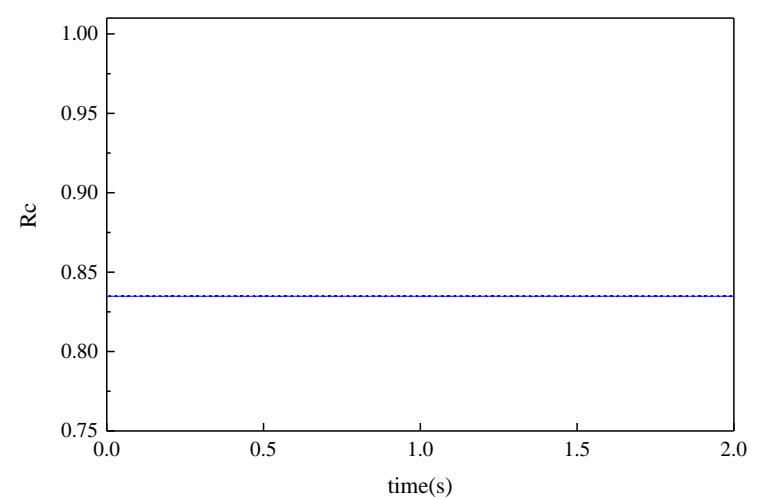

(a) $25 \mathrm{~Hz}$

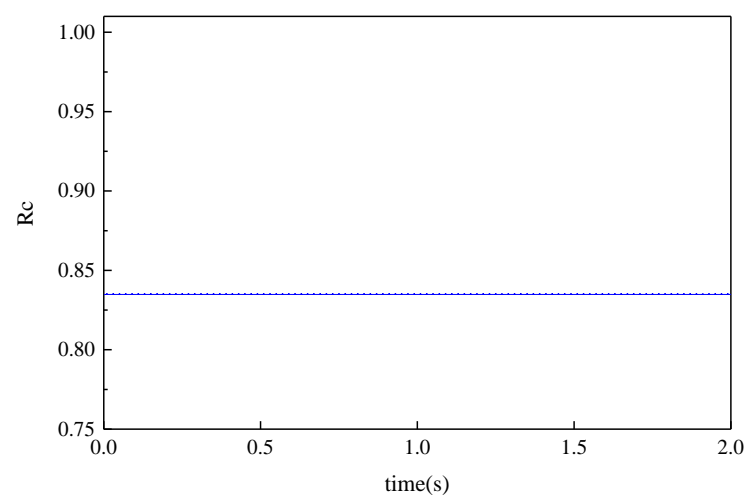

(b) $4725 \mathrm{~Hz}$

Fig. $11 \mathrm{Rc}$ of signals with $25 \mathrm{~Hz}$ perturbance and $4725 \mathrm{~Hz}$ perturbance $\left(a_{i}=0.3 a_{0}\right)$

\section{Experimental validation}

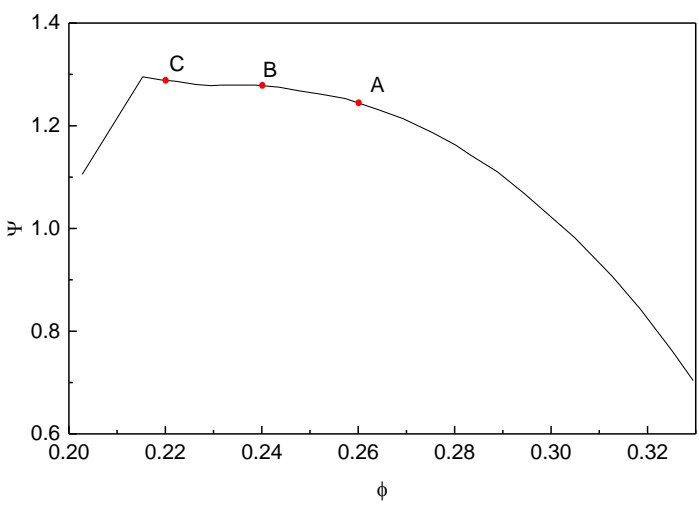

Fig.12 Pressure rise characteristics of 2-stage compressor

Based on the results of the signal simulation, we apply this stall warning approach on the two-stage compressor to validate whether this approach can provide sufficient warning time before the stall. Fig.12 gives the pressure rise characteristics of the 2-stage compressor at $100 \%$ design speed. The abscissa is flow coefficient $(\phi)$ and the ordinate represents for pressure rise coefficient $(\psi)$. In this figure, three points $\mathrm{A}, \mathrm{B}$, and $\mathrm{C}$ represent three different operation points from a large flow rate to near stall flow rate. The time traces of Rc at three points are illustrated in Fig.13. The timepressure data used here is collected from the leading edge of the first rotor. When the flow coefficient is 0.26 , the mean value of $\mathrm{Rc}$ is 0.827 . Then the work point moves to $\mathrm{B}$ where the flow coefficient is 0.24 and the mean value of $\mathrm{Rc}$ becomes 0.759. With further throttling, the mean value of Rc drops to 0.645 at the flow coefficient of 0.22 . According to Ref. [15], the cumulative distribution function can present the relationship between Rc and the stall margin more clearly. The cumulative distribution function of Rc can be expressed as

$$
F\left(R c_{t h}\right)=P\left(R c \leq R c_{t h}\right)
$$

where $\mathrm{Rc}_{\text {th }}$ is a given threshold of $\mathrm{Rc}$ and $\mathrm{P}(\cdot)$ is the probability. It describes the probability of Rc when it is lower than or equal to $\mathrm{Rc}_{\mathrm{th}}$. For instance, if a given sample of $\mathrm{Rc}$ spends $20 \%$ of its time below 0.9 (a setting threshold), the corresponding value of its distribution function $\mathrm{F}(0.9)$ would equal to 0.2 . The distribution function of Rc is displayed in Fig.14. Each curve corresponds to a different flow coefficient of the compressor. It can be seen in this figure that the distribution curves move left with the reduction of flow coefficient, which indicates if a proper $\mathrm{Rc}_{\mathrm{th}}$ is chosen, the value of $F\left(R c_{t h}\right)$ will grove with the reduction of stall margin. This changing tendency of $F\left(R_{t h}\right)$ has the potential to be used as a criterion of stall warning. According to the figure, the Rc stays beyond 0.6 almost all the time at operation point A while at operation $\mathrm{C}$, the $\mathrm{Rc}$ spends about one third of its time below 0.6. In detail, the values of $\mathrm{Rc}$ at points $\mathrm{A}, \mathrm{B}$ and $\mathrm{C}$ are $0.00675,0.07231$ and 0.3884 , respectively. Therefore, 0.6 is a feasible choice for $R c_{t h}$. Next, the warning limit of $F\left(R c_{t h}\right)$ should be determined. This stall warning approach will generate a warning signal the moment $F\left(R_{\text {th }}\right)$ reaches the warning limit. A lower warning limit may lead to a longer 
warning time, but it will also rise risks for false alarm. In this paper, the warning limit is 0.15 for the two-speed compressor to get sufficient warning time before stall boundary.

Time pressure traces of $\mathrm{Rc}$ and $\mathrm{F}\left(\mathrm{Rc}_{\mathrm{th}}\right)$ during a continuing throttling process are given in Fig.15. At the beginning of the throttling process, Rc stays at a relatively large level with some individual drops. As stall boundary comes closer, more and more sharp drops of Rc can be observed. The $\mathrm{Rc}_{\mathrm{th}}$ is set as 0.6 here. As shown in Fig.15, the value of $\mathrm{F}\left(\mathrm{Rc}_{\mathrm{th}}\right)$ increases almost monotonically with the decreasing of stall margin. If a warning limit is set as 0.15 , then this stall warning approach can generate a warning signal when the remain of stall margin is $5.5 \%(\phi=0.225)$. The time gap between generating a warning signal and stall point is 3.7 s (185 revolutions), which can provide sufficient time for modern devices to realize stability control for a compressor.

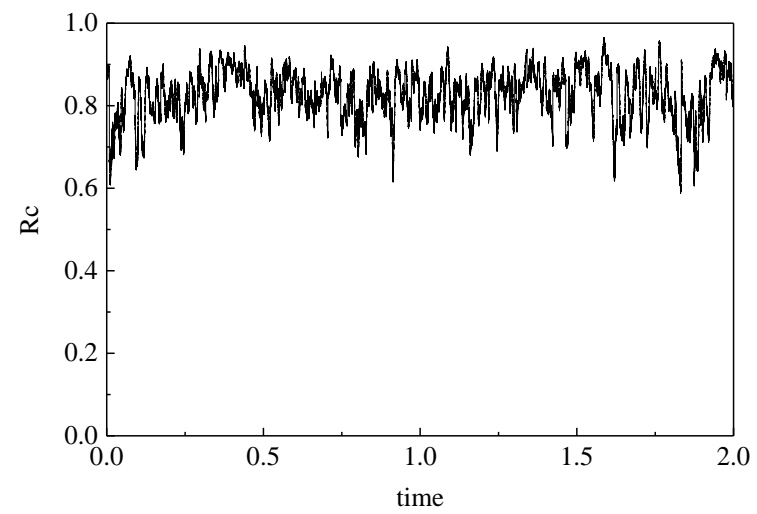

(a) $\phi=0.26$

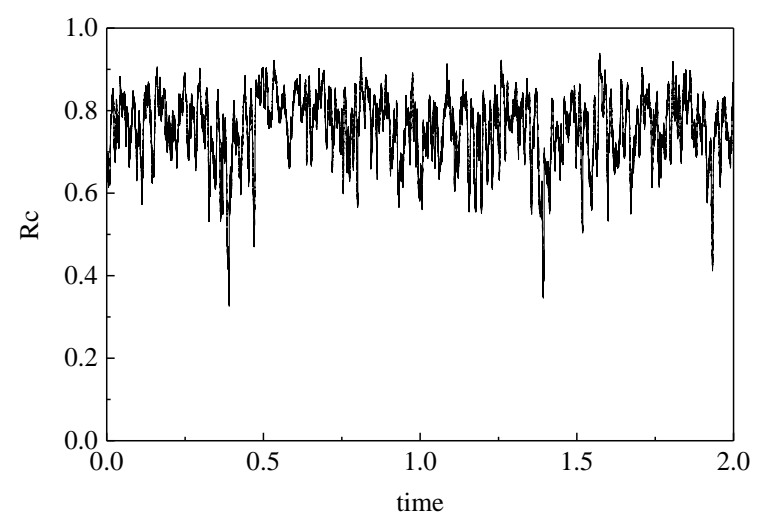

(b) $\phi=0.24$

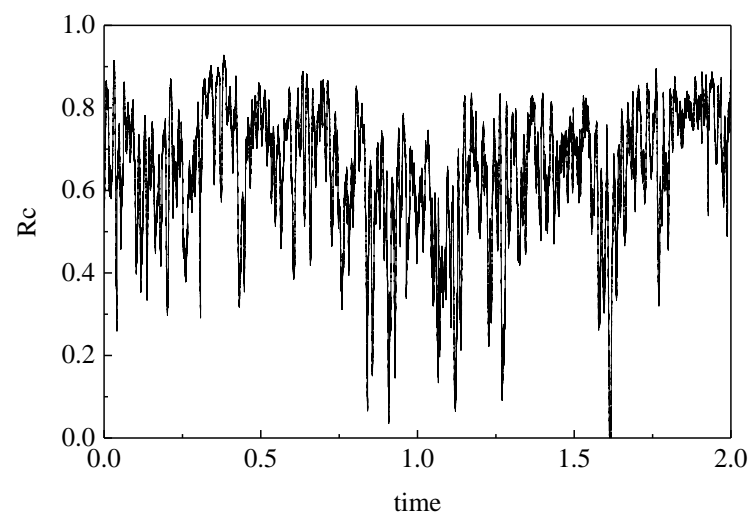

(c) $\phi=0.22$

Fig.13 Rc at different operation points

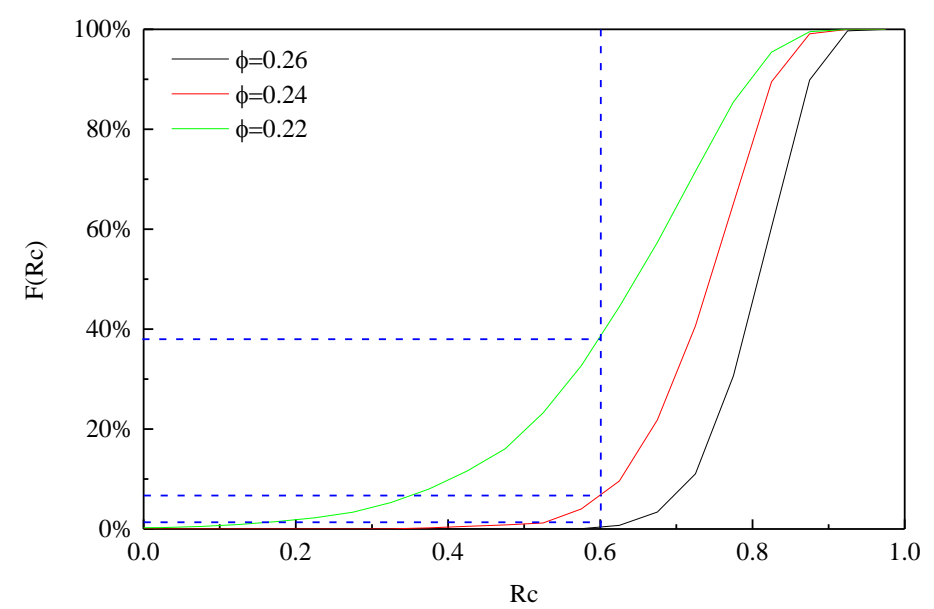

Fig.14 Distribution of Rc at different operation points

From the correlation measure results of signals during steady working points (Fig.13) and throttling process (Fig.15), it can be observed that at near stall point, the value of Rc is significantly lower and the range of Rc is relatively large. According to the results of signal simulation before, some low-frequency perturbance may exist and the power of the low frequency perturbance is amplified near stall boundary. To verify this, spatial Fourier analysis of signals from eight sensors distributed on the first stage is applied and Fig.16 depicts the time-resolved PSD results of the signals. The frequency here is normalized by the rotor speed and an evident low frequency perturbance can be identified in the figure. The perturbance emerges and grows several seconds before the stall. It appears that this perturbance may be responsible for the drop value of Rc. 


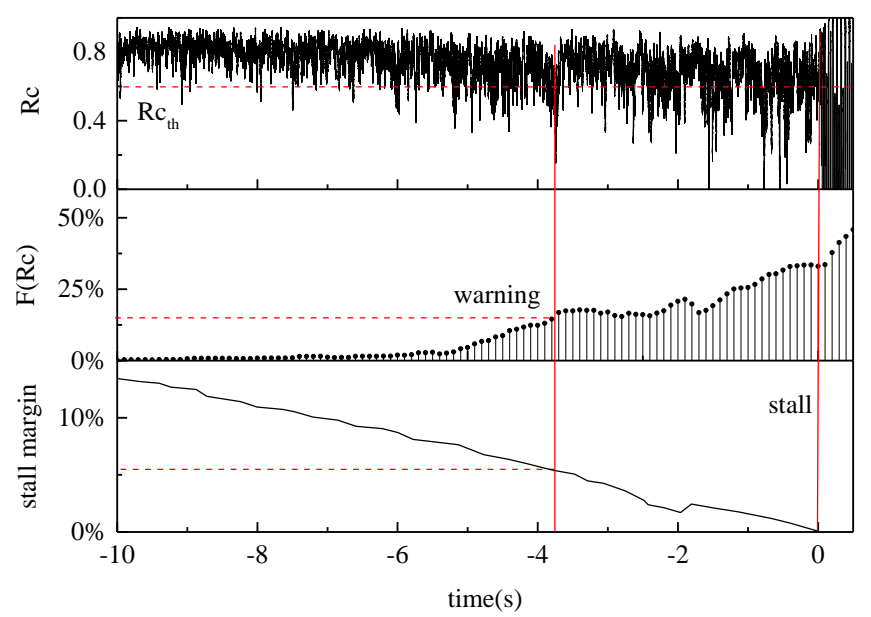

Fig.15 Experimental results on 2-stage compressor

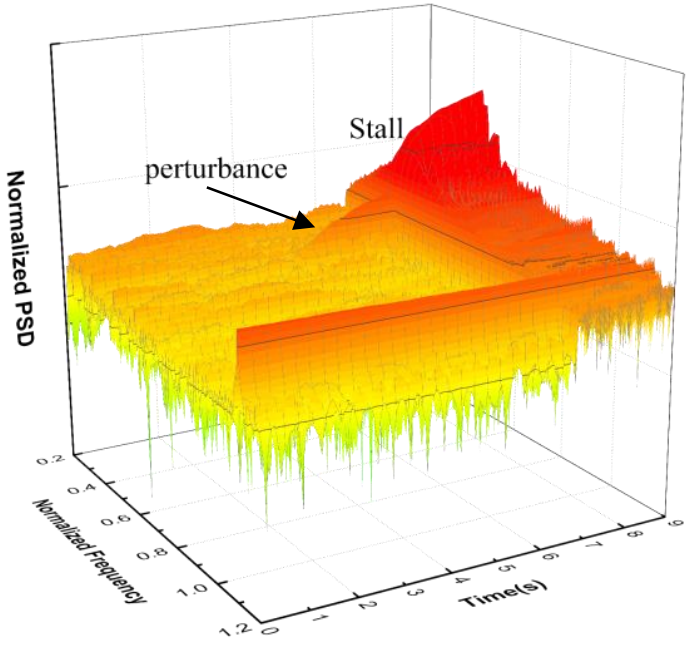

Fig.16 PSD results of stall evolution in the 2-stage compressor

\section{APPLICATION WITH SPS CASING TREATMENT}

In most cases, the distribution of perturbance in a multistage compressor is not uniform. Since the Rc can reflect the intensity of perturbance near the sensor position, this stall warning approach may be used to analyze the distribution of perturbance by using several sensors located at the different position of the compressor. Then a local control of perturbance can be utilized to realize stabilization of the compressor. This research uses the stall precursor-suppressed (SPS) casing treatment as a perturbance control device.

\section{SPS casing treatment}

The scheme of SPS casing treatment is shown in Fig.17, it is installed on the upstream side of the rotor blade with about one-half overlapped area on the blade tip region. The perforated ratio of this SPS casing treatment can be adjusted from 0 to $12 \%$, and there is an annular back-chamber in 50 $\mathrm{mm}$ height, $100 \mathrm{~mm}$ length. This figure also briefly shows the mechanism of SPS casing treatment: the tip-region flow comes in and out the back-chamber generating some vortexes shedding at the slot edge, and these vortexes shedding can interact with the perturbation pressure waves in the main flow, and this interaction can be recognized as a soft boundary condition on this system. The detailed information of SPS casing treatment can be founded in Ref. [17]. Fig. 18 shows the stability enhancement that SPS casing treatment brings to the two-stage compressor. The SPS casing treatment was installed on the first rotor. It can be seen in this figure that when the perforated ratio of SPS casing treatment is $12 \%$, it will bring the most significant improvement to the compressor stability. Thus, the SPS casing treatment with $12 \%$ perforated ratio is selected for the following experiments.
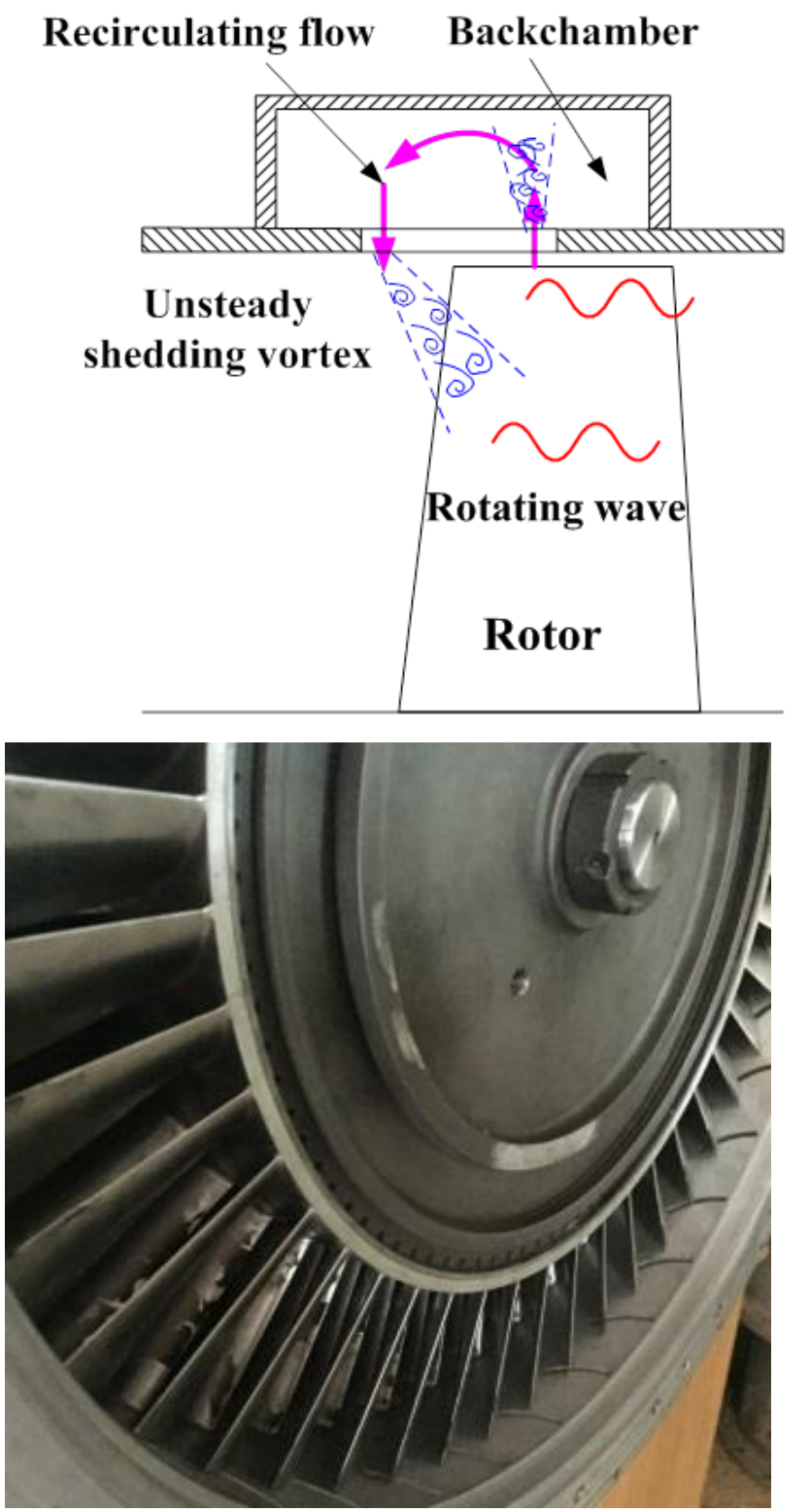

Fig. 17 SPS casing treatment schematic and installing status 


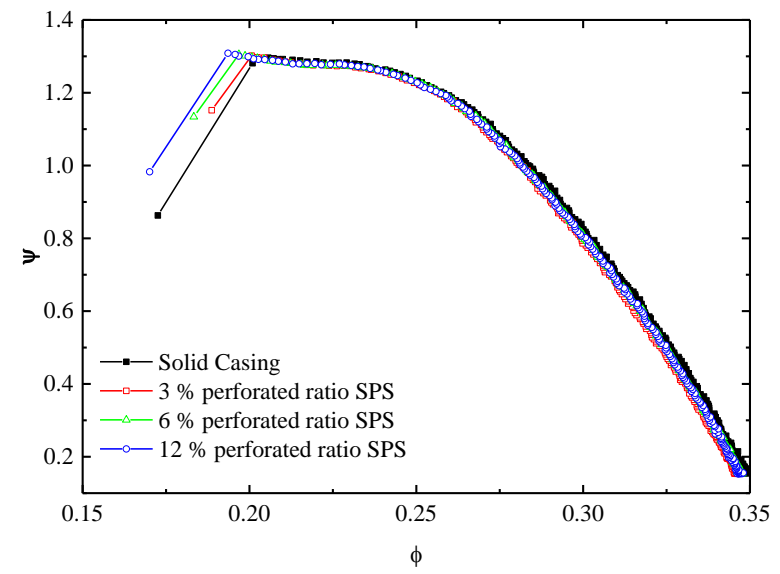

Fig. 18 SPS casing treatment with different perforated ratio

\section{Results from different axial position}

Firstly, the perturbance distribution at the different stage is analyzed. Fig. 19 shows the variance of Rc during the throttling process. The pressure data used in the Fig.19 is collected from sensors located at the leading edge of IGV, the first stage rotor, and the second stage rotor respectively. A downtrend of Rc with the reduction of stall margin can be found at all three axial locations. This result indicates that regardless of the signal is collected from what stage of this compressor, the stall warning approach is effective and can provide a warning signal seconds before the stall occurs. The Rc in Fig. 19a and Fig. 19c is higher with a slight decline trend, whereas the Rc in Fig.19b shows a noticeable decrease range near stall. It suggests that the intensity of pressure perturbation at the first stage is the most significant of all three axial locations. To evidence this, Fourier analysis is used here on the signals of the first stage and the second stage and the frequency characteristics of the signals are represented in Fig. 20. In this figure, some low frequency perturbance can be observed in both stages, and the amplitude of perturbance is much higher in the first stage than that in the second stage. Fig.21 displays the time-resolved signals of both two stages during the stall process. The normalized no-dimensional static pressure signals of 16 channels are shown in the same time axis. Some oblique lines in this figure show the revolution of the rotating stall. It is clear that the stall onset can be firstly found in the first stage, and the stall signals detected in the second stage are from the first stage. All these results mean that the perturbance is firstly generated at the first stage and then propagated to the second stage. As to the sensor located at the leading edge of IGV, it is far from the rotor and the blade-passing signal decays rapidly with the increase of the distance between sensor and rotor [16]. As a result, the perturbance in the blade-passing signal cannot have a significant influence on the signal before IGV, so the timepressure signal here maintains a good periodicity before stall occurs.

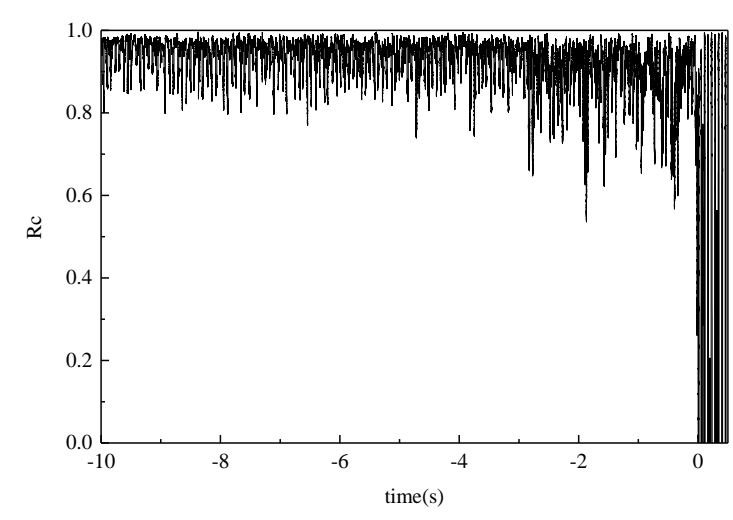

(a) Rc at the leading edge of IGV

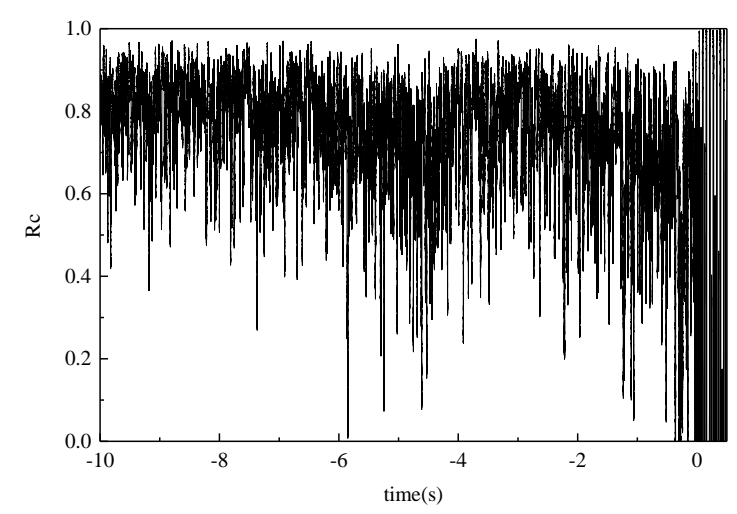

(b) Rc at the leading edge of the first rotor

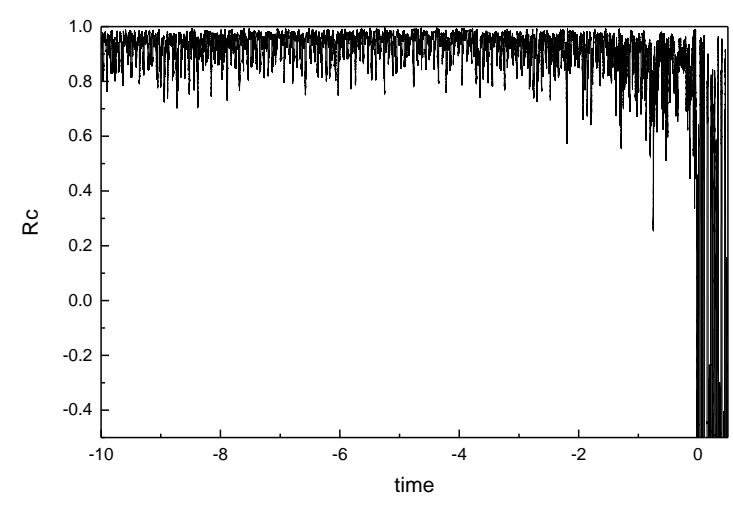

(c) Rc at the leading edge of the second rotor

Fig. 19 Rc at different position of the compressor

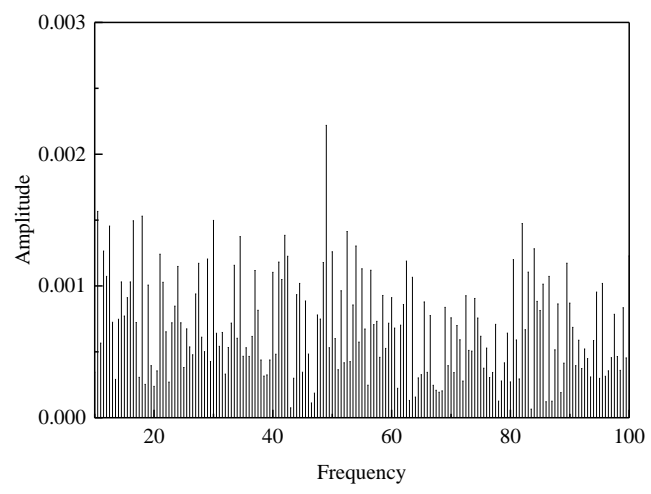

(a) first stage 


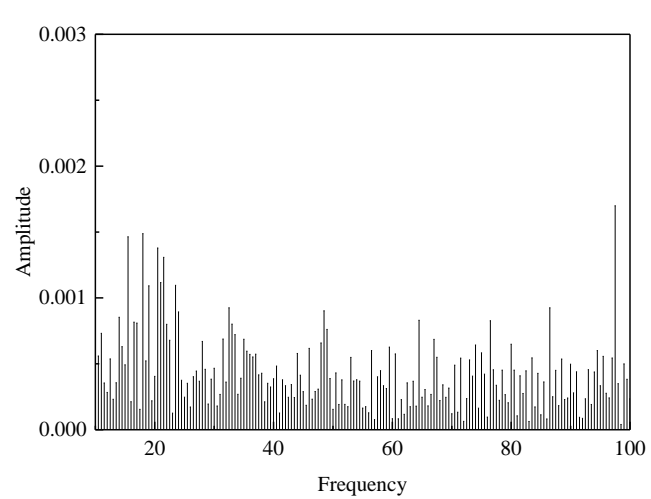

(b) second stage

\section{Fig.20 Frequency characteristics of stall evolution} in First-stage and Second-stage

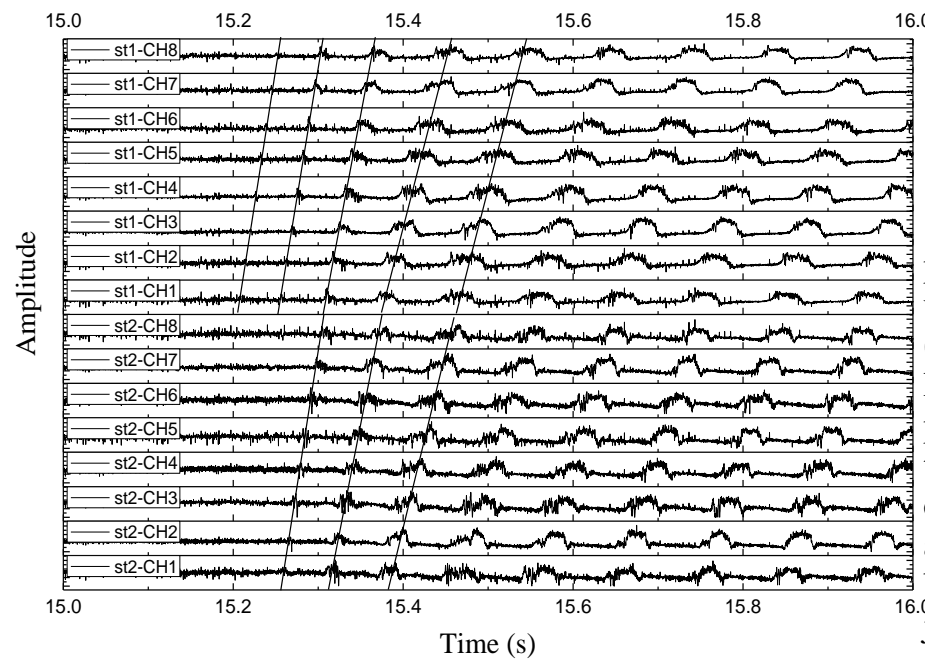

Fig. 21 Dynamic static pressure signals of stall evolution

According to the results above, the power of perturbance at the first stage is larger than the second stage, so if the SPS casing treatment is applied on the first stage, it will have a better effect on the compressor stability than on the second stage.

As evident in Fig.22, when SPS casing treatment is installed at the first stage, the improvement of stall margin is noticeable and almost identical with the case that SPS casing treatment is installed on both stages. However, if SPS casing treatment is only engaged on the second stage, it will not make a difference to stall margin compared with the solid casing. This is because the stall precursors emerged at first stage first, the SPS casing treatment at the first stage can suppress the growth of precursors and the SPS casing treatment at the second stage cannot influence the precursors at far field. The effects of stability enhancement of SPS casing treatment applied on the different stage are in good agreement with the result of perturbance distribution, which means the local control of perturbance is feasible in the multistage compressor.

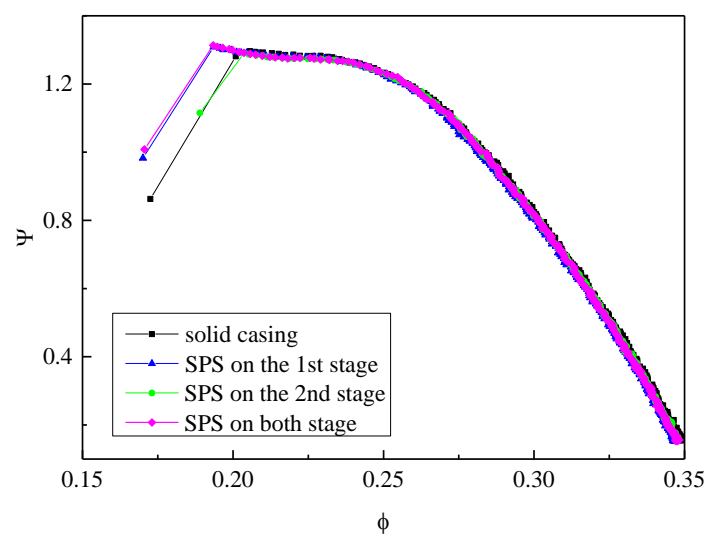

\section{Fig.22 Compressor performance lines of different casing conditions}

\section{Results from different circumferential positions}

To investigate the circumferential distribution of perturbance in the compressor, we use 8 sensors mounted uniformly around the casing at the leading-edge of IGV (Fig.2). Rc at different circumferential position is presented in Fig. 23. Sensors 1 to 4 show the degradation of Rc at near stall point where Rc of sensors 5 to 8 still keep a high level until the stall occurs. To see the variation of Rc distribution more clearly, $\mathrm{F}\left(\mathrm{Rc}_{\mathrm{th}}\right)$ is calculated (Fig.24). The value of $\mathrm{Rc}_{\mathrm{th}}$ is set as 0.75 . Indices of sensor 1 to 4 increase several seconds before stall while indices of sensors 5 to 8 stay near zero and just rise after stall.

The results of the correlation measure at different circumferential positions indicates that the perturbance mainly exists near the location of sensors 1 to 4 . It is mentioned that the perturbance is generated at the first stage. Based on this distribution of perturbance, part-circumference SPS casing treatment was applied on the first stage to verify the effect of this local control of the perturbance. The results of partial SPS casing treatment is provided in Fig.25. The operating range of compressor with lower half SPS casing treatment is similar to the compressor with whole casing treatment and it seems that only installing upper half SPS casing treatment will not bring obvious benefit to the compressor stability.

It can be concluded that in this two-stage compressor, perturbance mainly exists at the lower half of the first stage (area near the sensors 1 to 4) and this distribution of perturbance can be detected by the correlation measure of the pressure signal. Lower half SPS casing treatment can locally suppress the perturbance and extend the stable operating range of the compressor. 


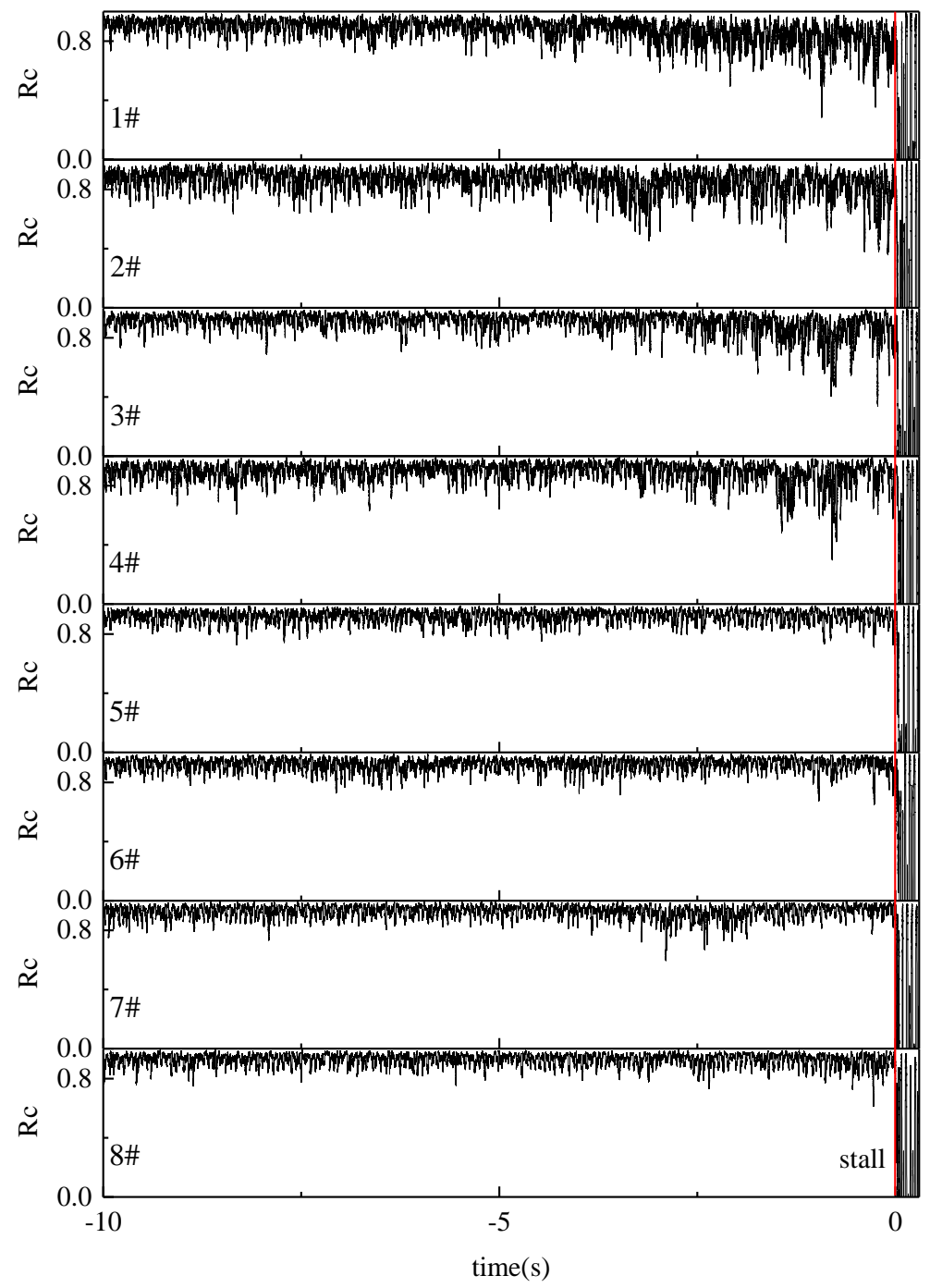

Fig.23 Rc at different circumferential positions 


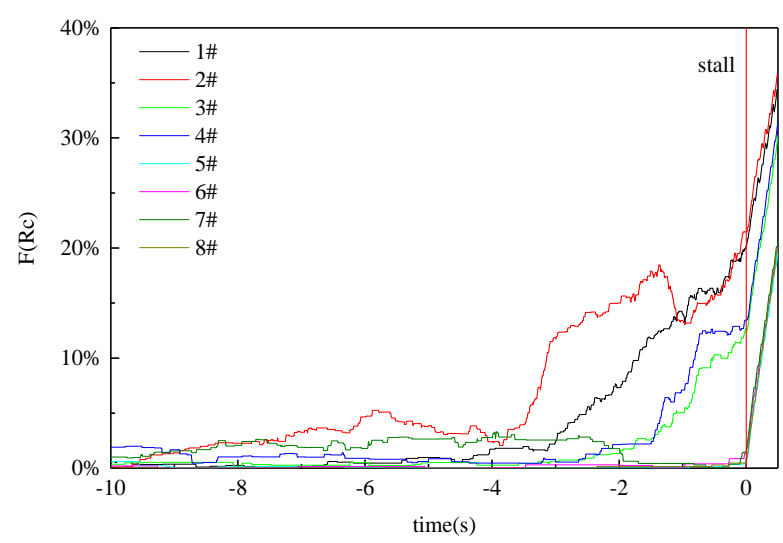

Fig.24 $F(R c)$ at different circumferential positions

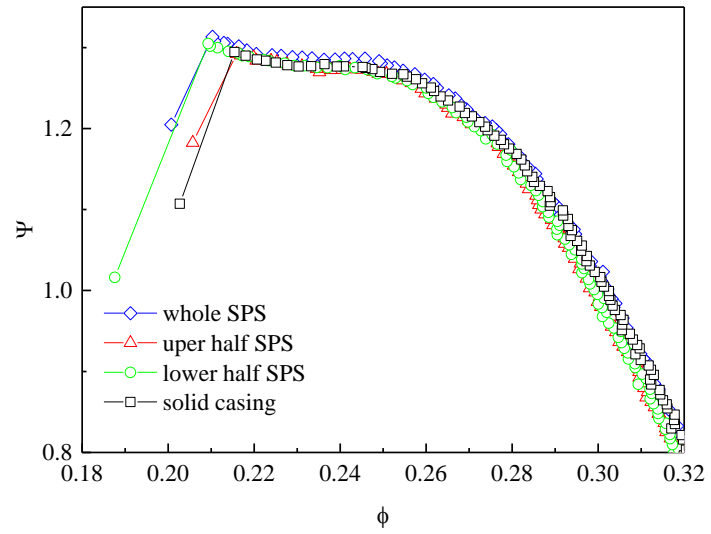

Fig.25 Compressor performance lines of different partial casing conditions

\section{CONCLUSION}

The main purpose of this study is to validate the effect of an acoustic-theory based stall warning approach and use this approach to detect the perturbance distribution in the compressor. Then based on the distribution of the perturbance, local control can be utilized with partial SPS casing treatment to extend the stable range of compressor. According to the results of signal simulation and experiments on the 2-stage compressor, conclusions can be drawn as follows:

The degree of Rc degradation can reflect the power of perturbance such as flow separation and vortex shedding in the compressor. When the stall boundary is close, the growth of perturbance power will cause a decrease of Rc. This feature can be used for stall warning to provide sufficient time for stability control.

Considering the spatial scale of flow separation and vortex shedding on blades, a length of three blades is a proper choice for the calculating window. This length of the calculating window can also help distinguish high frequency and low frequency perturbance.

In the two-stage compressor, this stall warning approach can be applied to both stages and provide a warning signal seconds before the stall. It can also be used to detect the distribution of the perturbance in the compressor. Thus this

stall warning approach can cooperate with other control devices such as casing treatment and air injection to realize a local control of compressor which can enhance the stability with lower cost.

Partial SPS casing treatment can be applied to the position where the power of perturbance is the most significant to suppress the perturbance regionally and enlarge the range of compressor effectively.

\section{ACKNOWLEDGMENTS}

The research presented here is supported by National Natural Science Foundation of China (Nos. 11661141020, 51576008, 51822601 and 51790514).

\section{NOMENCLATURE}

$a_{0}$

$a_{i}$

$f_{i}$

$f_{s}$

$F\left(R c_{t h}\right)$ the value of cumulative distribution function

$R c$

$n$

$N \quad$ the number of samples in one shaftperiod

$p_{0} \quad$ blade-passing signal

$p_{i} \quad$ perturbance signal

$p_{j}^{n} \quad$ a section of pressure signal

$P(\cdot) \quad$ probability

$R c_{t h} \quad$ the threshold of $R c$

$\phi \quad$ flow coefficient

$\Psi$

pressure rise coefficient 


\section{REFERENCES}

1. Epstein, A. H., Ffowcs Williams, J. E., and Greitzer, E. M. "Active suppression of aerodynamic instabilities in turbomachines," Journal of Propulsion and Power Vol. 5, No. 2, 1989, pp. 204-211.

doi: $10.2514 / 3.23137$

2. Williams, J. E. F., and Huang, X. Y. "Active stabilization of compressor surge," Journal of Fluid Mechanics Vol. 204, 1989, pp. 245-262.

doi: 10.1017/S0022112089001746

3. Day, I. J. "Stall Inception in Axial Flow Compressors," Journal of Turbomachinery Vol. 115, No. 1, 1993, pp. 1-9. doi: 10.1115/1.2929209

4. Garnier, V. H., Epstein, A. H., and Greitzer, E. M. "Rotating Waves as a Stall Inception Indication in Axial Compressors," No. 79047, 1990, p. V001T01A052.

doi: 10.1115/90-GT-156

5. Tryfonidis, M., Etchevers, O., Paduano, J. D., Epstein, A. H., and Hendricks, G. J. "Prestall Behavior of Several High-Speed Compressors," Journal of Turbomachinery Vol. 117, No. 1, 1995, pp. 62-80.

doi: $10.1115 / 1.2835644$

6. Höss, B., Leinhos, D., and Fottner, L. "Stall Inception in the Compressor System of a Turbofan Engine," Journal of Turbomachinery Vol. 122, No. 1, 1998, pp. 32-44.

doi: 10.1115/1.555425

7. Inoue, M., Kuroumaru, M., Tanino, T., and Furukawa, M. "Propagation of Multiple Short-Length-Scale Stall Cells in an Axial Compressor Rotor," Journal of Turbomachinery Vol. 122, No. 1, 1999, pp. 45-54.

doi: $10.1115 / 1.555426$

8. Liao, S., and Chen, J. "Time-Frequency Analysis of Compressor Rotating Stall by Means of Wavelet Transform," No. 78729, 1996, p. V001T01A013.

doi: 10.1115/96-GT-057

9. Inoue, M., Kuroumaru, M., Iwamoto, T., and Ando, Y. "Detection of a Rotating Stall Precursor in Isolated Axial Flow Compressor Rotors," Journal of Turbomachinery Vol. 113, No. 2, 1991, pp. 281-287.

doi: $10.1115 / 1.2929102$

10. Tahara, N., Kurosaki, M., Ohta, Y., Outa, E., Nakajima, T., and Nakakita, T. "Early Stall Warning Technique for AxialFlow Compressors," Journal of Turbomachinery Vol. 129, No. 3, 2006, pp. 448-456.

doi: $10.1115 / 1.2447948$

11. Tahara, N., Nakajima, T., Kurosaki, M., Ohta, Y., Outa, E., and Nisikawa, T. "Active stall control with practicable stall prediction system using auto-correlation coefficient," 37th Joint Propulsion Conference and Exhibit. American Institute of Aeronautics and Astronautics, 2001.

12. Dhingra, M., Neumeier, Y., Prasad, J. V. R., BreezeStringfellow, A., Shin, H.-W., and Szucs, P. N. "A Stochastic Model for a Compressor Stability Measure," No. 42371, 2006, pp. 833-843.

doi: $10.1115 /$ GT2006-91182

13. Dhingra, M., Neumeier, Y., Prasad, J. V. R., and Shin, H.-W. "Stall and Surge Precursors in Axial Compressors," 39th AIAA/ASME/SAE/ASEE Joint Propulsion Conference and Exhibit. American Institute of Aeronautics and Astronautics, 2003.

14. Young, A., Day, I., and Pullan, G. "Stall Warning by Blade Pressure Signature Analysis," No. 54679, 2011, pp. 1589-1599.

doi: $10.1115 / \mathrm{GT} 2011-45850$

15. Li, F., Li, J., Dong, X., Zhou, Y., Sun, D., and Sun, X. "Stall-Warning Approach Based on Aeroacoustic Principle," Journal of Propulsion and Power Vol. 32, No. 6, 2016, pp. 1353-1364.

doi: 10.2514/1.B35814

16. Dong, X., Li, F., Xu, R., Sun, D., and Sun, X. "Further Investigation on Acoustic Stall-Warning Approach in Compressors," Journal of Turbomachinery Vol. 141, No. 6, 2019, pp. 061001-061001-10.

doi: $10.1115 / 1.4041900$

17. Sun, D., Liu, X., Jin, D., Gui, X., and Sun, X. "Theory of Compressor Stability Enhancement Using Novel Casing Treatment, Part II: Experiment," Journal of Propulsion and Power Vol. 30, No. 5, 2014, pp. 1236-1247.

doi: 10.2514/1.B34901

18. Sun, D., Sun, X., Liu, X., Lin, F., and Qun, N. C. "Effect of Novel Casing Treatment on the Suppression of Stall Precursor in a Transonic Compressor," No. 45639, 2014, p. V02DT44A025.

doi: 10.1115/GT2014-26439

19. Sun, X., Sun, D., Liu, X., Yu, W., and Wang, X. "Theory of Compressor Stability Enhancement Using Novel Casing Treatment, Part I: Methodology," Journal of Propulsion and Power Vol. 30, No. 5, 2014, pp. 1224-1235.

doi: 10.2514/1.B34900

20. Dong, X., Sun, D., Li, F., Jin, D., Gui, X., and Sun, X. "Effects of Rotating Inlet Distortion on Compressor Stability With Stall Precursor-Suppressed Casing Treatment," Journal of Fluids Engineering Vol. 137, No. 11, 2015, pp. 111101111101-15.

doi: $10.1115 / 1.4030492$ 